\title{
INDICADORES DO ESTILO DE VIDA DOS DOCENTES DE UM CURSO DE EDUCAÇÃO FÍSICA
}

\author{
Claudia dos Passos Hartwig
}

Universidade Federal de Pelotas, Pelotas, Rio Grande do Sul, Brasil

\author{
Airton José Rombaldi \\ Universidade Federal de Pelotas, Pelotas, Rio Grande do Sul, Brasil \\ Marcelo Cozzensa da Silva \\ Universidade Federal de Pelotas, Pelotas, Rio Grande do Sul, Brasil
}

\begin{abstract}
Resumo
O presente estudo objetivou avaliar a frequência de hábitos alimentares saudáveis (FHAS) e o nível suficiente de prática de atividade física no lazer (NAFL) de professores de um curso superior em Educação Física do Rio Grande do Sul. Utilizouse o IPAQ longo para verificação do NAFL e os "10 passos da alimentação saudável" para a FHAS. A maioria dos docentes (66,7\%) foi considerada suficientemente ativa no lazer. Em relação à FHAS, o passo 8 foi o de maior adesão $(62,5 \%)$, seguido do passo 7 , com $41,7 \%$. Os passos com menor adesão foram o passo 5 , com $4,2 \%$, e os passos 4 e 2 , com $8,3 \%$ cada um. Nenhum professor aderiu a todos os passos. Concluiu-se que os docentes apresentam NAFL superior ao da população em geral, mas que há baixa adesão a hábitos de alimentação saudável.
\end{abstract}

Palavras-chave: Professores Universitários. Educação Física. Atividade Motora. Hábitos Alimentares.

\section{Introdução}

$\mathrm{H}$ á um crescente número de evidências (HACKSHAW; LAW; WALD, 1997; CRITCHLEY; UNAL, 2003; EYRE et al., 2004; BASSUK; MANSON, 2005; SOFI et al., 2008) de que o estilo de vida (baixos níveis de atividade física, hábito de fumar e má alimentação) é responsável por grande número de doenças, tais como obesidade, diabetes, hipertensão, infarto e alguns tipos de câncer. Além disso, a atividade ocupacional parece ser, também, importante fator de determinação de morbidades. Profissionais da educação, em especial professores, parecem estar mais expostos a doenças relacionadas a problemas vocais, lesões por movimentos repetitivos, alergias e trans- 
tornos emocionais (DELCOR et al., 2004; PORTO et al., 2004; GASPARIN, 2006).

Araújo et al. (2003) relatam que, no Brasil, existe uma escassez de estudos sobre a saúde do professor em comparação com trabalhadores de outras profissões. Na área de saúde ocupacional foram encontrados alguns estudos descritivos com professores que enfocam temas diversos como saúde mental (CODO, 2000), processo e condições de trabalho e repercussões sobre a saúde do educador (SILVANY-NETO et al., 2000; REIS et al., 2006) e acidentes de trabalho (MEDEIROS et al., 2005) entre outros.

Muito pouco se sabe, em relação a professores de Educação Física, sobre o comportamento de dois importantes fatores que podem contribuir para a prevenção de doenças crônicas: a prática regular de atividade física e os hábitos alimentares saudáveis. Em relação à prática de atividade física desses professores, poucos estudos foram realizados, pois parece haver uma espécie de senso comum que parte do pressuposto de que esse professor é naturalmente mais ativo que a população (PORTO et al., 2004; GASPARIN, 2006). Pesquisas realizadas na Europa relatam que professores de Educação Física apresentam níveis mais elevados de atividade física quando comparados a colegas que ministram outras disciplinas e a população em geral (PIHL; MATSIN; JURIMAE, 2002; RINTALA et al., 2003; SANDMARK, 2003). Em relação aos hábitos alimentares saudáveis a situação não é diferente, pois não foram localizados estudos na literatura sobre o tema.

Partindo dessa premissa, o presente estudo tem como objetivo avaliar a prática de atividade física no lazer e a adesão a uma dieta de qualidade e os fatores a elas associados em professores de um curso superior de Educação Física.

\section{Métodos}

Foi realizado um estudo transversal com a totalidade dos docentes $(n=24)$ de uma faculdade de Educação Física do estado do Rio Grande do Sul. Todos os participantes assinaram um termo de consentimento livre e esclarecido, e a pesquisa foi aprovada pelo Comitê de Ética da Universidade Federal de Pelotas (ESEF/UFPel) com número de protocolo 106/2009.

Características demográficas, socioeconômicas, comportamentais, nutricionais e de saúde foram avaliadas por meio de um questionário 
padronizado contendo 70 questões fechadas. A coleta de dados foi conduzida entre os meses de janeiro e março de 2010 por um entrevistador treinado. As medidas antropométricas foram conduzidas de acordo com a padronização sugerida por Gordon, Chumlea e Roche (1988).

As variáveis independentes foram categorizadas do seguinte modo: sexo (masculino ou feminino), idade ( $\leq 39,40-49$ e $\geq 50$ anos completos), tabagismo (nunca fumou, ex-fumante ou fumante atual), autopercepção de saúde (excelente, muito boa, boa/regular), área de pós-graduação (ciências biológicas/saúde, ciências sociais/humanas), índice de massa corporal (IMC), calculado a partir das medidas de peso e altura referidas (eutrófico, sobrepeso, obeso) e relação cinturaquadril (risco baixo, moderado, alto, muito alto). A classificação dos indivíduos seguiu as recomendações da Organização Mundial da Saúde (OMS) para o cálculo do IMC (WORLD HEALTH ORGANIZATION, 1995) e de Bray e Gray (1988) para os pontos de corte da relação cintura-quadril.

Os desfechos do estudo foram o nível suficiente de prática de atividade física no lazer, medida através do International Physical Activity Questionnaire (IPAQ), versão longa (CRAIG et al., 2003), e a frequência de hábitos alimentares saudáveis, utilizando como referência os "10 passos da alimentação saudável" (MINISTÉRIO DA SAÚDE, 2010) (Quadro 1).

Em relação aos domínios investigados pelo IPAQ, no presente estudo somente foi utilizado o domínio do lazer em uma semana habitual, na medida em que o nível de atividade física relatada nos ambientes do trabalho e doméstico parece ser superestimado (TEYCHENNE; BALL; SALMON, 2008; STAMATAKIS; HAMER; LAWLOR, 2009) e o domínio do lazer parece ser o mais lembrado (TIMPERIO; SALMON; CRAWFORD, 2003; STAMATAKIS; HAMER; LAWLOR, 2009).

Para a coleta dos dados sobre os passos relativos à alimentação foram utilizadas questões que permitiram verificar a frequência de consumo dos alimentos no período de um ano anterior à entrevista, segundo os "10 passos da alimentação saudável". Com base em cada um dos passos do documento do Ministério da Saúde, foram utilizadas questões simples, que permitiram uma resposta direta a cada um dos itens (VINHOLES; ASSUNÇÃO; NEUTZLING, 2009). Para os alimentos contidos na recomendação foi construído um quadro com uma 
pergunta inicial: "Pense sobre sua alimentação no último ano. Desde que [mês] do ano passado, com que frequência o(a) $\operatorname{Sr}(a)$ comeu cada um dos seguintes alimentos?” (VINHOLES; ASSUNÇÃO; NEUTZLING, 2009).

\begin{tabular}{|c|c|}
\hline Passo 1 & $\begin{array}{l}\text { Fazer pelo menos três refeições (café da manhã, almoço e jantar) e dois } \\
\text { lanches saudáveis por dia. Não pular as refeições. }\end{array}$ \\
\hline Passo 2 & $\begin{array}{l}\text { Incluir diariamente seis porções do grupo dos cereais (arroz, milho, trigo, } \\
\text { pães e massas), tubérculos, como as batatas, e raízes, como a mandioca, nas } \\
\text { refeições. Dar preferências aos grãos integrais e aos alimentos na sua forma } \\
\text { mais natural. }\end{array}$ \\
\hline Passo 3 & $\begin{array}{l}\text { Comer diariamente pelo menos três porções de legumes e verduras como } \\
\text { parte das refeições e três porções ou mais de frutas nas sobremesas e } \\
\text { lanches. }\end{array}$ \\
\hline Passo 4 & $\begin{array}{l}\text { Comer feijão com arroz todos os dias ou, pelo menos, cinco vezes por } \\
\text { semana. }\end{array}$ \\
\hline Passo 5 & $\begin{array}{l}\text { Consumir diariamente três porções de leite e derivados e uma porção de } \\
\text { carnes, aves, peixes ou ovos. Retirar a gordura aparente das carnes e a pele } \\
\text { das aves antes da preparação. }\end{array}$ \\
\hline Passo 6 & $\begin{array}{l}\text { Consumir, no máximo, uma porção por dia de óleos vegetais, azeite, } \\
\text { manteiga ou margarina. Ficar atento aos rótulos dos alimentos e escolher } \\
\text { aqueles com menores quantidades de gorduras trans. }\end{array}$ \\
\hline Passo 7 & $\begin{array}{l}\text { Evitar refrigerantes e sucos industrializados, bolos, biscoitos doces e } \\
\text { recheados, sobremesas e outras guloseimas como regra da alimentação. }\end{array}$ \\
\hline Passo 8 & $\begin{array}{l}\text { Diminuir a quantidade de sal na comida e retirar o saleiro da mesa. Evitar } \\
\text { consumir alimentos industrializados com muito sal (sódio) como } \\
\text { hambúrguer, charque, salsicha, linguiça, presunto, salgadinho, conservas de } \\
\text { vegetais, sopas, molhos e temperos prontos. }\end{array}$ \\
\hline Passo 9 & $\begin{array}{l}\text { Beber pelo menos } 2 \text { litros ( } 6 \text { a } 8 \text { copos) de água por dia. Dar preferência ao } \\
\text { consumo de água nos intervalos das refeições. }\end{array}$ \\
\hline Passo 10 & $\begin{array}{l}\text { Tornar a vida mais saudável. Praticar pelo menos } 30 \text { minutos de atividade } \\
\text { fisica todos os dias e evitar as bebidas alcoólicas e o fumo. Manter o peso } \\
\text { dentro dos limites saudáveis. }\end{array}$ \\
\hline
\end{tabular}

Quadro 1: Descrição dos "10 passos da alimentação saudável"

A seguir uma lista de alimentos foi lida ao entrevistado e a resposta marcada. Para isso, o questionário foi composto por questões de frequência de consumo alimentar de diferentes tipos de alimentos, o professor relatou o número de vezes que ingeriu o alimento e sua frequência correspondente (diária, semanal, mensal ou anual). Os passos coletados dessa forma foram os seguintes: passo 2 (cereais e grãos 
integrais), passo 3 (frutas, verduras e legumes), passo 4 (arroz com feijão), passo 5 (carnes, leites e derivados), passo 6 (óleos, margarina ou manteiga), passo 7 (doces, bolos, sucos e refrigerantes), passo 8 (alimentos industrializados, ricos em sal). Para contemplar inteiramente cada um dos passos, foram também utilizadas outras questões diretas, tais como: passo 5 ("Você costuma tirar a gordura aparente das carnes, a pele do frango ou outro tipo de ave?"), passo 6 ("Qual tipo de gordura é mais usada na sua casa para cozinhar os alimentos?", "Você costuma ler a informação nutricional que está presente no rótulo dos alimentos industrializados antes de comprá-los?"), passo 8 ("Você costuma colocar mais sal na sua comida quando seu prato já está servido?").

Os demais passos foram perguntados diretamente: passo 1 ("Quais dessas refeições você costuma fazer por dia") e passo 9 ("Quantos copos de água você toma por dia - água pura sem contar a incluída nos sucos, chá, chimarrão ou café?").

No passo 10, para avaliar o nível de atividade física, foi utilizado o IPAQ, versão longa. O escore foi calculado da seguinte forma: minutos por semana de caminhada + minutos por semana de atividade física moderada + (minutos por semana de atividade física vigorosa vezes 2) (US DEPARTMENT..., 2008). Foram considerados ativos os professores que atingiram 150 minutos de atividade física semanal e insuficientemente ativos aqueles que ficaram abaixo desse valor. Considerou-se fumante o professor que havia utilizado pelo menos um cigarro nos 30 dias anteriores. A avaliação do consumo de bebida alcoólica foi feita com uma questão sobre a frequência de consumo de bebidas no último ano (Com que frequência - vezes por dia/semana/mês/raramente ou nunca - você normalmente ingere bebidas alcoólicas como cerveja, vinho, uísque, conhaque, coquetéis ou outros), e foi considerada como positiva para a adesão parcial ao passo uma ingestão de bebidas de, no máximo, uma vez por semana. O Quadro 2 apresenta as categorias consideradas para a adesão a cada um dos passos.

Os dados foram digitados no programa Excel 2007 para Windows. A análise foi conduzida no programa estatístico STATA 10.0 utilizando os recursos da estatística descritiva (média, desvio-padrão [DP] para as variáveis contínuas e frequência relativa e intervalo de confiança de 95\% [IC95\%] para as variáveis categóricas). Para as medidas de associação entre as variáveis independentes e os desfechos categoriza- 
dos, utilizou-se o teste exato de Fisher. O nível de significância aceito foi de $\mathrm{p}<0,05$.

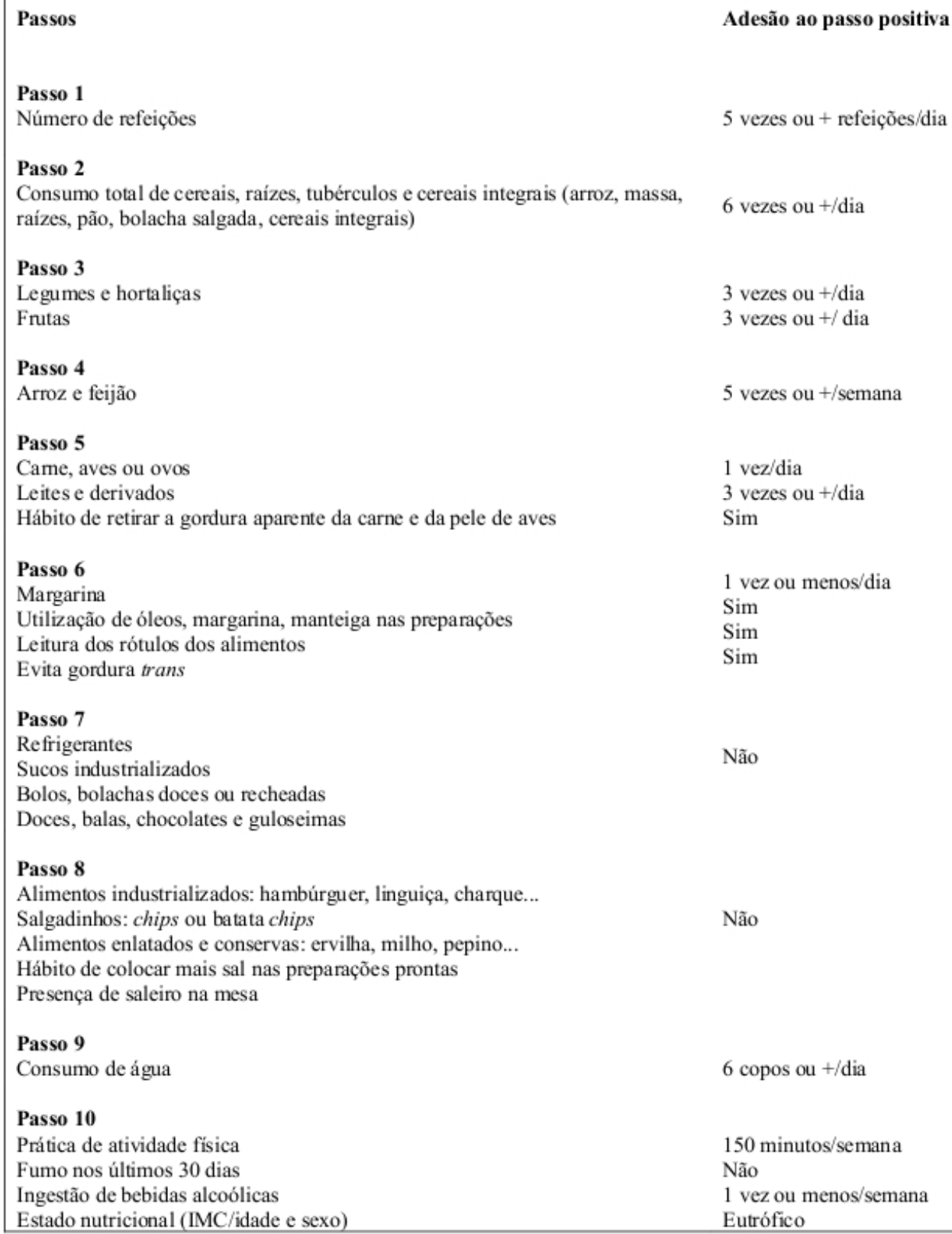

Quadro 2: Critérios utilizados para a adesão aos "10 passos da alimentação saudável" 


\section{Resultados}

Todos os docentes de Educação Física da instituição responderam ao questionário. A Tabela 1 descreve as características dos indivíduos em estudo. A maioria dos entrevistados era do sexo masculino $(70,8 \%)$ e apresentou média de idade de 46,3 (DP 9,2) anos. A maior parte dos docentes nunca havia fumado $(83,3 \%)$ e $41,7 \%$ consideravam sua saúde como excelente. Em relação ao estado nutricional (IMC), 11 professores foram classificados como eutróficos $(45,8 \%)$ e 10 foram considerados em risco para sobrepeso $(41,7 \%)$. Mais da metade dos docentes $(54,2 \%)$ apresentou risco moderado para doenças cardiovasculares e hipertensão, através da relação cintura-quadril, e $66,7 \%$ foram considerados suficientemente ativos no lazer. Em relação à área de formação na pós-graduação, $70,8 \%$ dos docentes referiram as ciências biológicas/saúde como a área mais procurada.

Tabela 1: Descrição da amostra de professores de Educação Física de ensino superior, Pelotas (RS), 2010 ( $n=24)$

\begin{tabular}{lll}
\hline Variável & $\mathrm{N}$ & $\%$ \\
\hline Sexo $\quad$ Masculino & 17 & 70,8 \\
Feminino & 7 & 29,2 \\
Idade (anos) & & \\
até 39 & 6 & 25,0 \\
$40-49$ & 9 & 37,5 \\
> 50 & 9 & 37,5 \\
Tabagismo & & \\
Nunca fumou & 20 & 83,3 \\
Ex-fumante & 3 & 12,5 \\
Fumante atual & 1 & 4,2 \\
Autopercepção de saúde & & \\
Excelente & 10 & 41,7 \\
Muito boa & 6 & 25,0 \\
Boa/regular & 8 & 33,3 \\
Área de pós-graduação & & \\
Biológicas/saúde & 17 & 70,8 \\
Humanas/ciências sociais & 7 & 29,2 \\
IMC (Kg/m ${ }^{2}$ Eúfico & & \\
Eutrófico & 11 & 45,8 \\
Sobrepeso & 10 & 41,7 \\
Obesidade & 3 & 12,5 \\
Relação cintura-quadril & & \\
Risco baixo & 2 & 8,3 \\
Risco moderado & 13 & 54,2 \\
Risco alto & 3 & 12,5 \\
Risco muito alto & 6 & 25,0 \\
Prática suficiente de atividade fisica no lazer & & 33,3 \\
Não & 16 & 66,7 \\
Sim & &
\end{tabular}


Na Tabela 2 está descrita a adesão aos " 10 passos da alimentação saudável". Nenhum dos professores alcançou todos os passos. O passo com maior frequência foi o de número 8 , no qual $62,5 \%$ dos respondentes relataram não comer alimentos industrializados e não acrescentar mais sal à refeição pronta. $\mathrm{O}$ passo com menor frequência foi o de número 5 , com adesão de apenas 1 entrevistado $(4,2 \%)$.

Tabela 2: Frequência dos "10 passos da alimentação saudável” em professores de Educação Física de ensino superior, Pelotas (RS), 2010 (n=24)

\begin{tabular}{|c|c|c|c|}
\hline 10 passos da alimentação saudável & $\mathrm{N}$ & $\%$ & $\mathrm{IC}_{95 \%}$ \\
\hline $\begin{array}{l}\text { Passo } 1 \text { - Faça pelo menos } 3 \text { refeições (café da manhã, } \\
\text { almoço e jantar) e } 2 \text { lanches saudáveis por dia. Não pule as } \\
\text { refeições. }\end{array}$ & 6 & 25,0 & $9,0-46,0$ \\
\hline $\begin{array}{l}\text { Passo } 2 \text { - Inclua diariamente } 6 \text { porções do grupo dos cereais } \\
\text { (arroz, milho, trigo, pães e massas), tubérculos, como as } \\
\text { batatas, e raízes, como mandioca/macaxeira/aipim nas } \\
\text { refeições. Dê preferência aos grãos integrais e aos alimentos } \\
\text { naturais. }\end{array}$ & 2 & 8,3 & $1,0-30,0$ \\
\hline $\begin{array}{l}\text { Passo } 3 \text { - Coma diariamente pelo menos } 3 \text { porções de } \\
\text { legumes e verduras como parte das refeições e } 3 \text { porções ou } \\
\text { mais de frutas nas sobremesas e lanches. }\end{array}$ & 3 & 12,5 & $3,0-30,0$ \\
\hline $\begin{array}{l}\text { Passo } 4 \text { - Coma feijão com arroz todos os dias ou, pelo } \\
\text { menos, } 5 \text { vezes por semana. }\end{array}$ & 2 & 8,3 & $1,0-30,0$ \\
\hline $\begin{array}{l}\text { Passo } 5 \text { - Consuma diariamente } 3 \text { porções de leite e } \\
\text { derivados e } 1 \text { porção de carnes, aves, peixes ou ovos. Retirar } \\
\text { a gordura aparente das carnes e a pele das aves antes da } \\
\text { preparação. }\end{array}$ & 1 & 4,2 & $1,0-20,0$ \\
\hline $\begin{array}{l}\text { Passo 6 - Consuma, no máximo, } 1 \text { porção por dia de óleos } \\
\text { vegetais, azeite, manteiga ou margarina. Fique atento aos } \\
\text { rótulos dos alimentos e escolha aqueles com menores } \\
\text { quantidades de gorduras trans. }\end{array}$ & 6 & 25,0 & $9,0-46,0$ \\
\hline $\begin{array}{l}\text { Passo } 7 \text { - Evite refrigerantes e sucos industrializados, bolos, } \\
\text { biscoitos doces e recheados, sobremesas doces e outras } \\
\text { guloseimas como regra da alimentação. }\end{array}$ & 10 & 41,7 & $22,0-63,0$ \\
\hline $\begin{array}{l}\text { Passo } 8 \text { - Diminua a quantidade de sal na comida e retire o } \\
\text { saleiro da mesa. Evite consumir alimentos industrializados } \\
\text { com muito sal (sódio) como hambúrguer, charque, salsicha, } \\
\text { linguiça, presunto, salgadinhos, conservas de vegetais, } \\
\text { sopas, molhos e temperos prontos. }\end{array}$ & 15 & 62,5 & $40,0-81,0$ \\
\hline $\begin{array}{l}\text { Passo } 9 \text { - Beba pelo menos } 2 \text { litros ( } 6 \text { a } 8 \text { copos) de água por } \\
\text { dia. Dê preferência ao consumo de água nos intervalos das } \\
\text { refeições. }\end{array}$ & 6 & 25,0 & $9,0-46,0$ \\
\hline $\begin{array}{l}\text { Passo } 10 \text { - Torne sua vida mais saudável. Pratique pelo } \\
\text { menos } 30 \text { minutos de atividade fisica todos os dias e evite as } \\
\text { bebidas alcoólicas e o fumo. Mantenha o peso dentro de } \\
\text { limites saudáveis. }\end{array}$ & 5 & 20,8 & $7,0-42,0$ \\
\hline Todos os passos & 0 & 0 & - \\
\hline
\end{tabular}


A relação entre as variáveis independentes e a frequência de adesão aos "10 passos da alimentação saudável" está na Tabela 3. Foi observada, no passo 1 , maior frequência de adesão das mulheres $(\mathrm{p}=0,04)$ e dos obesos $(\mathrm{p}=0,03)$. No passo 6 , fumantes atuais mostraram maior aderência quando comparados ao grupo dos que nunca fumaram $(\mathrm{p}=0,03)$. Quanto menor a idade e o IMC dos indivíduos, maior a frequência de adesão ao passo número 10.

Tabela 3: Frequências dos "10 passos da alimentação saudável” em professores de Educação Física de ensino superior, segundo variáveis independentes, Pelotas (RS), 2010

\begin{tabular}{|c|c|c|c|c|c|c|c|c|c|c|}
\hline Variáveis & $\begin{array}{c}\text { Pass } \\
\text { o } 1\end{array}$ & $\begin{array}{c}\text { Pass } \\
\text { o } 2\end{array}$ & $\begin{array}{c}\text { Pass } \\
\text { o } 3\end{array}$ & $\begin{array}{c}\text { Pass } \\
\text { o } 4\end{array}$ & $\begin{array}{c}\text { Pass } \\
\text { o } 5\end{array}$ & $\begin{array}{c}\text { Pass } \\
\text { o } 6\end{array}$ & $\begin{array}{c}\text { Pass } \\
\text { o } 7\end{array}$ & $\begin{array}{c}\text { Pass } \\
\text { o } 8\end{array}$ & $\begin{array}{c}\text { Pass } \\
\text { o } 9\end{array}$ & $\begin{array}{l}\text { Pass } \\
\text { o } 10\end{array}$ \\
\hline Sexo (valor $\mathrm{p})$ & $\begin{array}{c}0,04 \\
*\end{array}$ & 0,5 & 1,0 & 0,5 & 1,0 & 1,0 & 1,0 & 0,7 & 0,09 & 0,1 \\
\hline Masculino & 11,8 & 5,9 & 11,8 & 5,9 & 5,9 & 23,5 & 58,8 & 58,8 & 35,3 & 11,8 \\
\hline Feminino & 57,1 & 14,3 & 14,3 & 14,3 & 0 & 28,6 & 57,1 & 71,4 & 0 & 42,9 \\
\hline Idade (anos) (valor p) & 0,2 & 0,7 & 1,0 & 1,0 & 1,0 & 1,0 & 0,7 & 0,7 & 0,6 & $\begin{array}{l}0,04 \\
*\end{array}$ \\
\hline Até 39 & 0 & 16,7 & 16,8 & 0 & 0 & 33,3 & 66,7 & 83,3 & 33,3 & 50,0 \\
\hline $40-49$ & 44,4 & 11,1 & 11,1 & 11,1 & 0 & 22,2 & 44,4 & 55,6 & 11,1 & 22,2 \\
\hline$>50$ & 22,2 & 0 & 11,1 & 11,1 & 11,1 & 22,2 & 66,7 & 55,6 & 33,3 & 0 \\
\hline Tabagismo (valor $\mathrm{p}$ ) & 0,3 & 1,0 & 1,0 & 1,0 & 1,0 & $\begin{array}{c}0,03 \\
*\end{array}$ & 0,2 & 0,1 & 0,3 & 1,0 \\
\hline fumou Nunca & 20,0 & 10,0 & 15,0 & 10,0 & 5,0 & 15,0 & 50,0 & 70,0 & 20,0 & 25,0 \\
\hline Ex-fumante & 33,3 & 0 & 0 & 0 & 0 & 66,7 & 100,0 & 33,3 & 66,7 & 0 \\
\hline Fumante & 100,0 & 0 & 0 & 0 & 0 & 100,0 & 100,0 & 0 & 0 & 0 \\
\hline $\begin{array}{l}\text { Autopercepção de } \\
\text { saúde (valor p) }\end{array}$ & 0,3 & 0,3 & 0,2 & 0,3 & 1,0 & 1,0 & 0,5 & 1,0 & 0,9 & 0,09 \\
\hline Excelente & 40,0 & 20,0 & 30,0 & 20,0 & 10,0 & 30,0 & 50,0 & 60,0 & 20,0 & 20,0 \\
\hline Muito boa & 0 & 0 & 0 & 0 & 0 & 16,7 & 83,3 & 66,7 & 33,3 & 50,0 \\
\hline Boa/regular & 25,0 & 0 & 0 & 0 & 0 & 25,0 & 50,0 & 62,5 & 25,0 & 0 \\
\hline $\begin{array}{l}\text { Área de pós- } \\
\text { graduação (valor p) }\end{array}$ & 0,4 & 1,0 & 0,5 & 1,0 & 0,7 & 0,6 & 0,6 & 1,0 & 1,0 & 0,5 \\
\hline Riolóoicas'caúde & 29,4 & 11,8 & 17,7 & 11,8 & 5,9 & 29,4 & 58,8 & 64,7 & 23,5 & 23,5 \\
\hline $\begin{array}{l}\text { Humanas/ciências } \\
\text { sociais }\end{array}$ & 14,3 & 0 & 0 & 0 & 0 & 14,3 & 57,1 & 57,1 & 28,6 & 14,3 \\
\hline IMC (valor p) & $\begin{array}{c}0,03 \\
*\end{array}$ & 0,6 & 0,3 & 0,6 & 0,5 & 0,8 & 1,0 & 0,6 & 1,0 & $\begin{array}{c}0,03 \\
*\end{array}$ \\
\hline Eutrófico & 36,4 & 18,2 & 27,3 & 18,2 & 0 & 27,3 & 54,5 & 63,6 & 27,3 & 45,5 \\
\hline Sobrepeso & 0 & 0 & 0 & 0 & 10,0 & 30,0 & 60,0 & 70,0 & 20,0 & 0 \\
\hline Obesidade & 66,8 & 0 & 0 & 0 & 0 & 0 & 66,7 & 33,3 & 33,3 & 0 \\
\hline $\begin{array}{l}\text { Relação cintura- } \\
\text { quadril (valor p) }\end{array}$ & 1,0 & 1,0 & 0,3 & 0,3 & 1,0 & 1,0 & 0,1 & 0,3 & 0,5 & 0,2 \\
\hline Risco baixo & 0 & 0 & 50,0 & 50,0 & 0 & 0 & 0 & 100,0 & 0 & 0 \\
\hline Risco & 23,1 & 7,7 & 7,7 & 7,7 & 7,8 & 23,1 & 76,9 & 46,2 & 38,5 & 38,5 \\
\hline moderado & & & & & & & & & & \\
\hline Risco alto & 33,3 & 0 & 0 & 0 & 0 & 33,3 & 33,3 & 100,0 & 0 & 0 \\
\hline Risco muito & 33,3 & 16,8 & 16,7 & 0 & 0 & 33,3 & 50,0 & 66,7 & 16,7 & 0 \\
\hline $\begin{array}{l}\text { Prática suficiente de } \\
\mathrm{AF} \text { (valor } \mathrm{p} \text { ) }\end{array}$ & 0,6 & 1,0 & 0,5 & 1,0 & 0,3 & 1,0 & 0,3 & 0,4 & 0,5 & 0,3 \\
\hline Não & 33,3 & 0 & 0 & 0 & 16,7 & 16,7 & 83,3 & 50,0 & 16,7 & 0 \\
\hline Sim & 22,2 & 11,1 & 16,7 & 11,1 & 0 & 27,8 & 50,0 & 66,7 & 27,8 & 27,8 \\
\hline
\end{tabular}

* teste exato de Fisher

Pensar a Prática, Goiânia, v. 15, n. 2, p. 272-550, abr.jjun. 2012 
A Tabela 4 apresenta a relação entre atividade física e as variáveis independentes. A única variável associada ao desfecho foi o estado nutricional (IMC), em que 82,0\% dos indivíduos mostraram-se suficientemente ativos no lazer.

Tabela 4: Associação entre prática suficiente de atividade física no lazer e as variáveis independentes do estudo $(n=24)$

\begin{tabular}{|c|c|c|c|c|c|}
\hline & \multicolumn{4}{|c|}{$\begin{array}{l}\text { Prática suficiente de atividade física no } \\
\text { lazer }\end{array}$} & \multirow[t]{3}{*}{ Valor $\mathrm{p}$} \\
\hline & \multicolumn{2}{|c|}{ Sim } & \multicolumn{2}{|c|}{ Não } & \\
\hline & $\mathrm{n}$ & $\%$ & $\mathrm{~N}$ & $\%$ & \\
\hline Sexo & & & & & 0,6 \\
\hline Masculino & 12 & 70,6 & 5 & 29,5 & \\
\hline Feminino & 4 & 57,1 & 3 & 42,9 & \\
\hline Idade (anos) & & & & & 0,9 \\
\hline até 39 & 4 & 66,7 & 2 & 33,3 & \\
\hline $40-49$ & 7 & 77,8 & 2 & 22,2 & \\
\hline$>50$ & 5 & 55,6 & 4 & 44,4 & \\
\hline Tabagismo & & & & & 0,8 \\
\hline Nunca fumou & 13 & 65,0 & 7 & 35,0 & \\
\hline Ex-fumante & 2 & 67,0 & 1 & 33,0 & \\
\hline Fumante atual & 1 & 100 & 0 & 0 & \\
\hline Autopercepção de saúde & & & & & 0,6 \\
\hline Excelente & 7 & 70,0 & 3 & 30,0 & \\
\hline Muito boa & 5 & 83,3 & 1 & 16,7 & \\
\hline Boa/regular & 4 & 50,0 & 4 & 50,0 & \\
\hline Área de pós-graduação & & & & & 0,4 \\
\hline Biológica/saúde & 10 & 58,8 & 7 & 41,2 & \\
\hline Humanas/ciências sociais & 6 & 85,7 & 1 & 14,3 & \\
\hline $\operatorname{IMC}\left(\mathrm{Kg} / \mathrm{m}^{2}\right)$ & & & & & $0,03 *$ \\
\hline Eutrófico & 9 & 81,8 & 2 & 18,2 & \\
\hline Sobrepeso & 7 & 70,0 & 3 & 30,0 & \\
\hline Obesidade & 0 & 0,0 & 3 & 100,0 & \\
\hline Relação cintura-quadril & & & & & 1,0 \\
\hline Risco baixo & 2 & 100,0 & 0 & 0,0 & \\
\hline Risco moderado & 10 & 76,9 & 3 & 26,1 & \\
\hline Risco alto & 1 & 33,3 & 2 & 66,7 & \\
\hline Risco muito alto & 3 & 50,0 & 3 & 50,0 & \\
\hline
\end{tabular}

* teste exato de Fisher 


\section{Discussão}

O presente estudo realizou um levantamento de indicadores de estilo de vida de professores do ensino superior de um curso da área da saúde. Propôs-se a avaliar a prática suficiente de atividade física no tempo de lazer e a frequência da adesão à estratégia dos "10 passos da alimentação saudável” para a promoção de hábitos saudáveis de alimentação em adultos, segundo proposta do Ministério da Saúde do Brasil. Destaca-se que, para conhecimento dos autores, não foi encontrado outro estudo algum com o mesmo enfoque. Assim como houve dificuldade na comparação dos desfechos com outros estudos, também houve dificuldade em avaliar o consumo alimentar (WILLET, 1998; KAC; SICHIERI; GIGANTE, 2007), na medida em que se assumiu através da frequência de alimentação o consumo de porções adequadas.

Corroborando os resultados de estudo recente (VINHOLES; ASSUNÇÃO; NEUTZLING, 2009), as mulheres comportaram-se diferentemente dos homens em relação ao passo número 1 (pelo menos três refeições e dois lanches/dia), apresentando maior aderência. No entanto, apenas $25 \%$ dos entrevistados aderiram, de forma semelhante ao que foi encontrado em estudo de base populacional que incluiu adultos da cidade de Pelotas (MADRUGA; ARAUJO; BERTOLDI, 2009). Também observou-se associação entre a adesão a esse passo e o estado nutricional, sendo que os obesos aderiam mais ao passo que aqueles com sobrepeso e eutróficos. Provavelmente, esse resultado é consequência da estratégia de medida adotada para quantificar o número de porções adequado, o qual foi realizado através da medida da frequência alimentar.

No passo 6 (consumir no máximo uma porção de óleos, vegetais, azeite, manteiga ou margarina/dia) encontrou-se diferença significativa entre fumantes e não fumantes quanto à aderência ao passo. Verificou-se que o fumante aderiu ao passo, enquanto apenas $15 \%$ dos não fumantes o fizeram. Supõe-se que os sujeitos fumantes, já preocupados com sua condição de saúde, tentem compensar o hábito negativo do tabagismo com a adoção de uma alimentação correta. Esse achado deve ser visto com cuidado, na medida em que somente um entrevistado assumiu ser tabagista.

Docentes mais jovens e com menor IMC aderiram mais ao passo 10 (prática de atividade física diária, não fumar e não ingerir bebidas 
alcoólicas; manter o peso). $\mathrm{O}$ fato de os mais jovens e eutróficos serem mais ativos já foi demonstrado em outros estudos de base populacional conduzidos em Pelotas (HALLAL et al., 2003; HALLAL; SIQUEIRA, 2004; HALLAL et al., 2005) e os docentes não se diferenciaram da população em que estão inseridos.

Em relação à prática suficiente de atividade física no lazer, os respondentes do presente estudo se mostraram muito mais ativos no lazer (66,7\%) que professores de Educação Física do ensino básico $(55,9 \%)$ (CANABARRO; NEUTZLING; ROMBALDI, 2011) e que a totalidade da população $(30,2 \%)$ (ROMBALDI et al., 2010) da cidade de Pelotas. Ao analisar os poucos estudos internacionais com professores de Educação Física, os resultados mostram prevalências de prática suficiente de atividade física semelhantes aos do presente estudo e, seguindo na mesma direção, mostram que essas prevalências são superiores nesses docentes do que na população em geral (PIHL; MATSIN; JURIMAE, 2002; RINTALA et al., 2003). É provável que profissionais de Educação Física tenham mais conhecimento acerca dos benefícios da prática regular do exercício físico que a população, sendo, portanto, mais ativos no lazer.

Quanto à associação entre prática suficiente de atividade física no lazer com as variáveis independentes, a única que apresentou associação foi o estado nutricional. Esse resultado é coerente com os achados de estudos recentes realizados em adultos da zona urbana da cidade de Pelotas (HALLAL et al., 2003; HALLAL; SIQUEIRA, 2004; HALLAL et al., 2005). Estudo de Neutzling et al. (2009) demonstrou que adultos mais ativos no lazer consomem mais frutas, legumes e verduras, resultado corroborado pelos achados do presente estudo.

A baixa prevalência de adesão aos " 10 passos da alimentação saudável" pode ser explicada parcialmente pela dificuldade em atender os muitos critérios colocados em cada passo, de forma que a não observância de um critério inviabiliza a aderência ao passo completo. Nesse sentido, os passos 2 (incluir seis porções de cereais/dia), 3 (ingerir pelo menos três porções de legumes, verduras e frutas), 5 (consumir três porções de leite/derivados e uma porção de carne/dia), 6 (consumir no máximo uma porção de óleos, vegetais, azeite, manteiga ou margarina/dia), 7 (evitar consumo de refrigerantes, bebidas açucaradas, doces e guloseimas), 8 (diminuir a quantidade de sal) e 10 (prática de atividade física diária, não fumar e não ingerir bebidas alcoólicas, manter o peso) apresentam inúmeros critérios. Devido a isso, outros estudos re- 
alizados com esse instrumento têm relatado baixas prevalências de adesão (MADRUGA; ARAUJO; BERTOLDI, 2009; VINHOLES; ASSUNÇÃO; NEUTZLING, 2009).

Concluiu-se que os docentes de um curso superior de Educação Física apresentaram prática de atividade física superior à da população em geral. No entanto, ao contrário do que se esperaria, a prevalência de prática de atividade física no lazer não alcança $2 / 3$ da totalidade dos professores. Os hábitos alimentares dos docentes são preocupantes, tendo em vista a baixa adesão à alimentação saudável. Considerando que o curso de Educação Física se insere na área da saúde, o comportamento alimentar e o hábito de atividade física são importantes temas da formação dos estudantes desse curso. As prevalências insuficientes dos desfechos estudados podem implicar em menores ênfases durante o curso de formação.

\title{
Life style aspects of physical education college teachers
}

\begin{abstract}
The aims of this study were to evaluate the frequency of healthy eating habits (FHEH) and sufficient level of leisure time physical activity (LTPA) in Physical Education college teachers from Rio Grande do Sul. A long version of IPAQ was accessed to verify the level LTPA and the "10 Steps to Healthy Feeding" to attend FHEH. Most teachers (66.7\%) were considered sufficiently active during leisure time. Regarding the FHEH, step 8 showed the highest adherence $(62.5 \%)$, followed by step 7 , with $41.7 \%$. Lower adherence was found to step 5 with $4.2 \%$ and steps 4 and 2 , with $8.3 \%$ each. No teacher adhered to all the steps. It was concluded that teachers have higher LTPA than the general population, but there is poor adherence to healthy eating habits.
\end{abstract}

Keywords: Faculty. Physical Education. Motor Activity. Food Habits.

Indicadores de los estilos de vida de los maestros de una facultad de educación física

\section{Resumen}

El estudio tuvo como objetivos evaluar la frecuencia de los hábitos alimentares saludables (FHLS) y el nivel suficiente de actividad física en el tiempo libre (NATL) de profesores licenciados de una facultad de Educación Física en Río Grande do Sul. Fue utilizado el IPAQ - versión larga para verificar el NATL y los "10 Pasos para una Alimentación Saludable" para medir la FHLS. La mayoría de los profesores $(66,7 \%)$ se consideraron suficientemente activos en el ocio. En cuanto a FHLS, el paso 8 fue el de más alta adherencia (62,5\%), seguido por el paso 7 , con un $41,7 \%$. Los pasos con baja adherencia fueran el paso 5 con $4,2 \%$ y los pasos 4 y 2 , con un $8,3 \%$. Ninguno maestro se ha adherido a todos los pasos. Se concluyó que 
los profesores han NATL superior que la población general, pero hay falta de adherencia a hábitos alimentares saludables.

Palabras clave: Profesores Universitários. Educación Física. Actividad Motora. Hábitos Alimentares.

\section{Referências}

ARAÚJO, T. M. et al. Saúde e trabalho docente: dando visibilidade aos processos de desgaste e adoecimento docente a partir da construção de uma rede de produção coletiva. Educação em Revista, Belo Horizonte, v. 37, p. 183-212, 2003.

BASSUK, S. S.; MANSON, J. E. Epidemiological evidence for the role of physical activity in reducing risk of type 2 diabetes and cardiovascular disease. Journal of Applied Physiology, Washington, v. 99, n. 3, p. 1193-1204, 2005.

BRAY, G. A.; GRAY, D. S. Obesity. Part I. Pathogenesis. Western Journal of Medicine, San Francisco, v. 149, n. 4, p. 429-441, 1988.

CANABARRO, L. K.; NEUTZLING, M. B.; ROMBALDI, A. J. Nível de atividade física no lazer dos professores de Educação Física do ensino básico. Revista Brasileira de Atividade Física e Saúde, Londrina, v. 16, n. 1, p. 11-17, 2011.

CODO, W. Educação: carinho e trabalho: burnout, a síndrome da desistência do educador, que pode levar à falência da educação. Rio de Janeiro: Vozes, 2000.

CRAIG, C. L. et al. International physical activity questionnaire: 12country reliability and validity. Medicine and Science in Sports and Exercise, Madison, v. 35, n. 8, p. 1381-1395, 2003.

CRITCHLEY, J. A.; UNAL, B. Health effects associated with smokeless tobacco: a systematic review. Thorax, London, v. 58, n. 5, p. 435443, 2003.

DELCOR, N. S. et al. Labor and health conditions of private school teachers in Vitória da Conquista, Bahia, Brazil. Cadernos de Saúde Pública, Rio de Janeiro, v. 20, n. 1, p. 187-196, 2004. 
EYRE H. et al. Preventing cancer, cardiovascular disease, and diabetes: a common agenda for the American Cancer Society, the American Diabetes Association, and the American Heart Association. Circulation, Dallas, v. 109, n. 25, p. 3244-3255, 2004.

GASPARIN, S. M. Prevalência de transtornos mentais comuns em professores da rede municipal de Belo Horizonte, Minas Gerais, Brasil. Cadernos de Saúde Pública, Rio de Janeiro, v. 22, n. 12, p. 26792691, 2006.

GORDON, C. C.; CHUMLEA, W. C.; ROCHE, T. G. Stature, recumbent length, and weight. In: LOHMAN, T. G.; ROCH, A. F.; MARTORELL, R. Anthropometric standardization reference manual. Champaign: Human Kinetics, 1988. p. 3-8.

HACKSHAW, A. K.; LAW, M. R.; WALD, N. J. The accumulated evidence on lung cancer and environmental tobacco smoke. British Medical Journal, London, v. 315, n. 7114, p. 980-988, 1997.

HALLAL, P. C. et al. Physical inactivity: prevalence and associated variables in Brazilian adults. Medicine and Science in Sports and Exercise, Madison, v. 35, n. 11, p. 1894-1900, 2003.

HALLAL, P. C.; SIQUEIRA, F. V. Compliance with vigorous physical activity guidelines in Brazilian adults: prevalence and correlates. Journal of Physical Activity and Health, Champaign, v. 1, p. 389397, 2004.

HALLAL, P. C. et al. Physical activity in adults from two Brazilian areas: similarities and differences. Cadernos de Saúde Pública, Rio de Janeiro, v. 21, n. 2, p. 573-580, 2005.

KAC, G.; SICHIERI, R.; GIGANTE, D. P. Epidemiologia nutricional. Rio de Janeiro: Editora Fiocruz, 2007.

MADRUGA, S. W.; ARAUJO, C. L. P.; BERTOLDI, A. D. Frequência do consumo de alimentos fontes de fibras e fatores associados em população do Sul do Brasil. Cadernos de Saúde Pública, Rio de Janeiro, v. 25, p. 2249-2259, 2009.

MEDEIROS, M. A. T. et al. Acidentes de trabalho em Piracicaba em 2002. Saúde em Revista, Piracicaba, v. 7, n. 15, p. 7-14, 2005. 
MINISTÉRIO DA SAÚDE. Coordenação Geral da Política de Alimentação e Nutrição. Alimentação saudável para todos: siga os 10 passos. Disponível em: <http://www.nutricao.saude.gov.br/documentos/10passos_adultos.pdf $>$.Acesso: em 26 jan. 2010.

NEUTZLING, M. et al. Fatores associados ao consumo de frutas, legumes e verduras em adultos de uma cidade no sul do Brasil. Cadernos de Saúde Pública, Rio de Janeiro, v. 25, n. 11, p. 2365-2374, 2009.

PIHL, E.; MATSIN, T.; JURIMAE, T. Physical activity, musculoskeletal disorders and cardiovascular risk factors in male physical education teachers. Journal of Sports Medicine and Physical Fitness, Torino, v. 42, n. 4, p. 466-471, 2002.

PORTO, L. A. R. et al. Doenças ocupacionais em professores atendidos pelo Centro de Estudos da Saúde do Trabalhador. Revista Baiana de Saúde Pública, Salvador, v. 28, n. 1, p. 33-49, 2004.

REIS, E. J. F. B. et al. Docência e exaustão emocional. Educação \& Sociedade, Campinas, v. 27, n. 94, p. 229-253, 2006.

RINTALA, P. et al. Physical activity and breast cancer risk among female physical education and language teachers: a 34-year follow-up. International Journal of Cancer, New York, v. 107, n. 2, p. 268-270, 2003.

ROMBALDI, A. J. et al. Leisure-time physical activity: association with activity levels in other domains. Journal of Physical Activity and Health, Champaign, v. 7, p. 460-464, 2010.

SANDMARK, H. Musculoskeletal dysfunction in physical education teachers. Occupational and Environmental Medicine, London, v. 57, n. 10, p. 673-677, 2003.

SILVANY-NETO, A. et al. Condições de trabalho e saúde dos professores da rede particular de ensino de Salvador. Revista Baiana de Saúde Pública, Salvador, v. 24, n. 3-4, p. 42-56, 2000.

SOFI, F. et al. Physical activity during leisure time and primary prevention of coronary heart disease: an updated meta-analysis of cohort 
studies. European Journal of Cardiovascular Prevention and Rehabilitation, London, v. 15, n. 3, p. 247-257, 2008.

STAMATAKIS, E.; HAMER, M.; LAWLOR, D. A. Physical activity, mortality, and cardiovascular disease: is domestic physical activity beneficial? The Scottish Health Survey - 1995, 1998, and 2003. American Journal of Epidemiology, Baltimore, v. 169, n. 10, p. 1191-1200, 2009.

TEYCHENNE, M.; BALL, K.; SALMON, J. Associations between physical activity and depressive symptoms in women. International Journal of Behavioral Nutrition and Physical Activity, London, v. 5, p. 27, 2008.

TIMPERIO, A.; SALMON, J.; CRAWFORD, D. Validity and reliability of a physical activity recall instrument among overweight and non-overweight men and women. Journal of Science and Medicine in Sport, Belconnen, v. 6, n. 4, p. 477-491, 2003.

US DEPARTMENT OF HEALTH AND HUMAN SERVICES. Physical activity guidelines for Americans. 2008. Disponível em: $<$ http://www.health.gov/paguidelines/pdf/paguide.pdf $>$. Acesso em: 29 mar. 2009.

VINHOLES, D. B.; ASSUNÇÃO, M. C. F.; NEUTZLING, M. B. Frequência de hábitos saudáveis de alimentação medidos a partir dos 10 passos da alimentação saudável do Ministério da Saúde: Pelotas, Rio Grande do Sul, Brasil. Cadernos de Saúde Pública, Rio de Janeiro, v. 25, n. 4, p. 791-799, 2009.

WILLET, W. C. Nutritional epidemiology. New York: Oxford University Press, 1998.

WORLD HEALTH ORGANIZATION. Physical status: the use and interpretation of anthropometry. Report of WHO Expert Committee. Geneva: World Health Organization, 1995. (WHO Technical Report Series, 854).

Recebido em: 23/04/2011

Revisado em: 08/07/2011

Aprovado em: 10/10/2011

Pensar a Prática, Goiânia, v. 15, n. 2, p. 272-550, abr./jun. 2012 


\section{Endereço para correspondência}

rombaldi@ufpel.tche.br

Airton José Rombaldi

Universidade Federal de Pelotas

Escola Superior de Educação Física, Departamento de Desportos.

Rua Luís de Camões, 625

Três Vendas

96055-630 - Pelotas, RS - Brasil 